\title{
Electroencephalographic Abnormalities in Patients with Idiopathic Insomnia
}

\author{
Debora Yoaly Arana Lechuga ${ }^{1}$, Oscar Sánchez Escandón ${ }^{1}$, Miguel Collado Corona ${ }^{2}$, \\ Carlos Castillo Montoya ${ }^{1}$, González Rosa Robles ${ }^{3}$, Anabel Jiménez Anguiano ${ }^{4}$, \\ Oscar Prospero García ${ }^{5}$, Javier Velázquez Moctezuma ${ }^{1}$
}

\begin{abstract}
${ }^{1}$ Sleep Disorders Clinic, Universidad Autónoma Metropolitana-Iztapalapa, Mexico City, Mexico; ${ }^{2}$ American British Cowdray Medical Center's Neurophysiology Department, Mexico City, Mexico; ${ }^{3}$ Mathematic Department, Universidad Autónoma MetropolitanaIztapalapa, Mexico City, Mexico; ${ }^{4}$ Neuroscience, Universidad Autónoma Metropolitana-Iztapalapa, Mexico City, Mexico; ${ }^{5}$ Neuroscience Laboratory, Medical School, Universidad Nacional Autónoma de México, Mexico City, Mexico.

Email: ctds@xanum.uam.mx
\end{abstract}

Received April 15 $5^{\text {th }}$ 2011; revised May 24 $4^{\text {th }}, 2011$; accepted July 15 $5^{\text {th }}, 2011$.

\begin{abstract}
Objective: The main goal of the present research is to describe the electroencephalographic activity during sleep and the cyclic alternating pattern (CAP) on idiopathic insomniac patients. Methods: 22 idiopathic insomnia patients were included. Sleep recording included: fully conventional EEG recording (32 electrodes, with 16 channels getting ipsilateral bipolar recordings), EOG, EMG, EKG, thoracic and abdominal respiratory movements, nasal flow and oximetry. Results: Out of the 22 registered patients, 10 (45\%) showed no electroencephalographic alteration, while 12 (55\%) exhibited an electroencephalographic alteration (irritative activity). Concerning PSG variables, significant differences between groups (no EEG alteration and EEG alterations) were detected in: sleep latency, total sleep time, total wake time, sleep efficiency, light sleep percentage, slow waves sleep percentage and on the sub type A1 index of the cyclic alternant pattern CAP. Conclusions: The present data strongly suggest that within the population diagnosed with idiopathic insomnia, there is a subgroup in which the electroencephalographic alterations may play a critical role as the source of the sleep abnormality.
\end{abstract}

Keywords: Irritative Activity, Idiopathic Insomnia, Cyclic Alternating Pattern

\section{Introduction}

Insomnia is the inability to initiate or maintain, as much as the subjective impression of not having a restorative sleep [1]. This debilitating medical condition affects negatively the patients' daily performance. Among many other consequences, the patient suffers of cognitive, such as attention and memory, and emotional alterations [2]. They also become much more vulnerable to illnesses [3]. Insomnia is the most common reported disorder in the sleep clinic [4]. In this kind of patients, clinic evaluation is critical. When, as a result of this evaluation, another sleep disorder or another medical condition is detected and it is found as the cause of the insomnia, this disorder is defined by the American Psychiatric Association (APA) in the DSM-IV, as secondary insomnia. Otherwise is called primary insomnia [5]. In this context it is noteworthy to mention that, the international classification of sleep disorders catalogs three types of primary insomnia: the psychophysiological, the paradoxical and the idiopathic insomnia [1].

The idiopathic insomnia is characterized by its long time-span; frequently the idiopathic insomnia patients complain about sleeping poorly "all the time"; and to have some exacerbation and remission periods. As above mentioned, this type of insomnia must not be related to another illness that could be causing the insomnia; it must not be either related to pharmacologic treatment or drug misuse [1].

During a polysomnographic study in patients with insomnia, the following findings have been described: increased sleep-onset latency and total wake time. Electroencephalographic activations such as an increase of the number of awaking have also been reported [6]; however, other authors did not find these alterations [7]. Lafranchi et al. recorded a group of patients with primary insomnia during three consecutive nights, reporting a decrease in total sleep time (411 minutes), in the sleep efficiency 
$(85 \%)$ and in the slow wave sleep percentage $(8 \%)$. Besides, they found also an increment of wake time after sleep onset (WASO) (60 minutes); however, the sleep latency remained unchanged [8]. In addition, a recent study carried out in Mexican population with primary insomnia, reported a reduction in total sleep time (382 minutes), along with a decreased of sleep efficiency $(78.8 \%)$ [9]. Interestingly, changes in the Cyclic Alternating Pattern (CAP) were described in those patients. The CAP is an electroencephalographic pattern characterized by a sudden change of voltage and frequency in the background electroencephalographic activity during No REM sleep (NREM). Once present, the pattern should appear rhythmically at least two times. Each CAP is constituted by a phase $\mathrm{A}$, that refers to the changes on the electroencephalographic activity (synchronization or desynchronization) that stands out of the background activity and a phase $\mathrm{B}$, that is the background activity again, $i$. $e$. the same voltage and frequency occurring before the beginning of phase A. Furthermore, there are three subtypes of phase A: synchronized activity (A1), mixed activity (A2) or desynchronized activity (A3). It has been suggested that subtype A1 is a promoter of sleep continuity, whereas subtypes A2 and A3 are promoters of the central nervous system activation [10]. Previous studies have described an increase of CAP A1 and A2 idiopathic insomnia patients [11].

On the other hand, a conventional PSG study provides only limited information about electroencephalographic activity [12]. Electrodes setting in a standard PSG study includes only six channels with monopolar derivations. This kind of setting is unable to detect potential electroencephalographic alterations.

Despite the different approaches by which idiopathic insomnia has been studied, up to date there is not yet solid evidence concerning its etiology. Electroencephalographic hyper excitability has been described in this kind of patients $[13,14]$. Another study has described an increment of corticosteroids and adrenaline [15]. Using PET studies, Nofzinger reported an increase of cerebral glucose metabolism in insomniac patients during sleepwake transition [16].

Therefore, it is possible that the presence of idiopathic insomnia is correlated with the presence of abnormal brain activity, which could only be detected using a complete EEG electrode setting. The main goal of the present research is to describe the electroencephalographic activity during sleep on idiopathic insomniac patients.

\section{Method}

Patients were recruited from different sleep disorders centers during their first medical evaluation. In the first step, diagnosis was clinically confirmed by at least two professionals, including one of our center and another from a different sleep clinic. In addition, patients were fully informed about the study and the signature of the informed consent was obtained. Patients agreed to delay any kind of treatment or therapy to relieve their sleep disorder until the sleep recording was performed. The sleep recordings were obtained in our Sleep Disorders Clinic, using 32 channels, Cadwell digital polygraph. All the studies started about $22.30 \mathrm{hrs}$ pm and finished at 06.30 hrs am. Immediately after the study, patients returned to their own Sleep Disorder Center to start their personalized treatment. Patients in whom another sleep disorder was detected with the sleep recording, for example: movement sleep disorder or breathing abnormalities during sleep were excluded from the final analysis.

22 patients $($ males $=8$; females $=14$ ) were included in the final analysis, showing an age range between 19 and 45 years. Sleep recording included: Fully conventional EEG recording (32 electrodes, with 16 channels getting ipsilateral bipolar recordings), electrooculography (EOG), electromyography (EMG) and electrocardiographic (EKG) recordings as well as respiratory variables such as thoracic and abdominal respiratory movements, nasal flow and oximetry.

Scoring of sleep recordings was carried out by following the Rechtschaffen and Kales guidelines [17], and its recent adaptations [18]. CAP detection was done by following the criteria published in 2007 [10].

Two independent neurophysiologists who were unaware of the patients' clinical history defined the electroencephalographic abnormalities. Patients were divided into two groups based on the presence or absence of electroencephalographic abnormalities. Statistical significance was obtained by using a $\mathrm{T}$ test.

\section{Results}

Concerning PSG variables, all the subjects included showed data compatible with the diagnosis of insomnia. The parameter affected in all the subjects was sleep efficiency (mean $72.5 \% \pm 15.3 \%$ ). $81.8 \%$ percent of subjects showed an increase in sleep latency (mean \pm SEM 40.7 $\min \pm 33.6$ ). $100 \%$ of subjects showed increase of Wake time after sleep onset (WASO) (mean \pm SEM $92.3 \mathrm{~min} \pm$ 61.3). Table 1 showed the data concerning PSG parameters obtained compared to those reported for normal sleepers within the same group of age.

As mentioned above, the presence of electroencephalographic alterations allow us to divide our population in two groups. Out of the 22 registered patients, $10(45 \%)$ showed no electroencephalographic alteration (Group A), while $12(55 \%)$ exhibited an electroencephalographic alteration (Group B). Table 2 summarizes the findings concerning differences between groups. The mean age of group A was 31 years, whereas the mean age of group B 
Table 1. Polysomnographic parameters: shows means and standard error (SEM) of polysomnographic variables in idiopathic insomniac compare with those reported for normal sleepers.

\begin{tabular}{ccc}
\hline & Insomnia patients $(22)$ & Control \\
\hline SL min $( \pm$ SEM $)$ & $40.7(33.6)$ & $10-20$ \\
TST min $( \pm$ SEM $)$ & $348.1(74.5)$ & \\
TWT $\%( \pm$ SEM $)$ & $27.5(15.4)$ & $\leq 10 \%$ \\
WASO min $( \pm$ SEM) & $92.3(61.3)$ & $\leq 10 \%$ \\
REML min $( \pm$ SEM $)$ & $120.6(59.2)$ & $\leq 90-120$ \\
LS\% $( \pm$ SEM $)$ & $67.2(9.3)$ & $60 \%$ \\
SWS $\%( \pm$ SEM $)$ & $16.3(8.1)$ & $20 \%$ \\
REM $\%( \pm$ SEM $)$ & $16.4(4.4)$ & $20 \%$ \\
Awakenings num $( \pm$ SEM) & $18(16.6)$ & $\leq 5$ \\
Arousal Index $( \pm$ SEM $)$ & $9.8(7.8)$ & $\leq 5$ \\
SE $( \pm$ SEM $)$ & $72.5(15.3)$ & $\geq 90 \%$ \\
CAP Index A1 $( \pm$ SEM) & $77.2(36.5)$ & 34.7 \\
CAP Index A2 $( \pm$ SEM $)$ & $23.6(11)$ & 12.8 \\
CAP Index A3 $( \pm$ SEM $)$ & $41.8(6.2)$ & 9.4 \\
\hline
\end{tabular}

Table 2. Differences between group: shows the means of the clinical features between patients with electroencephalographic abnormalities and without them.

\begin{tabular}{ccc}
\hline & Group A & Group B \\
& $\mathrm{N}=10$ & $\mathrm{~N}=12$ \\
\hline Age (years) & 31 & 28 \\
Gender & $\mathrm{M} 2$ & $\mathrm{M} 6$ \\
proportion & $\mathrm{W} 8$ & $\mathrm{~W} 6$ \\
Evolving time (year) & 16.5 & 6 \\
Age of onset & 15 & 22 \\
\hline
\end{tabular}

was 28 years. The evolving time of the disorder was longer in group A. Men $(50 \%)$ and women $(50 \%)$ were equally represented in group B while in group A men $(20 \%)$ were underrepresented as compared with women $(80 \%)$. It is noteworthy to mention that the proportion of men with insomnia and electroencephalographic alteration was $75 \%$ while females barely reached $42.8 \%$.

Regarding the electroencephalographic alterations, their expression was restricted to the left hemisphere in $66 \%$ of the patients, while the remaining $33 \%$ of the patients expressed them in both left and right hemispheres (Table 3). $75 \%$ of the patients showed an alteration localized in the frontal lobe, $16 \%$ in the central-frontal re gion and the remaining $9 \%$ on the central-parietal region.

Out of 12 patients in the group B, $8(66 \%)$ exhibited abnormalities in the left brain hemisphere, while the rest of them $(4,33 \%)$ presented them in both hemispheres.

The most frequent electroencephalographic alterations was: sharp waves in the $83 \%$ of the patients and phase inversion spike in $28 \%$ of the patients. Spikes and slow wave activity was also observed in $16 \%$ of the patients (Table 3). It must be noticed that some patients presented more than one electroencephalographic alteration. (Figure 1).
Table 3. Electroencephalographic abnormalities: shows percentage of patients who presented electroencephalographic abnormalities during sleep and its location.

\begin{tabular}{lcc}
\hline \multicolumn{1}{c}{ EEG alteration/location } & Number $(\mathrm{N}=12)$ & $\%$ \\
\hline Sharp-waves & 10 & 83 \\
Sharp-wave activity with phase in version & 4 & 28 \\
Spikes & 2 & 16 \\
Slow Wave Activity & 2 & 16 \\
Left & 8 & 66.6 \\
Right & 0 & 0 \\
Bila teral & 4 & 33.3 \\
\hline
\end{tabular}

Concerning PSG variables, significant differences between groups were detected in: sleep latencies $(p<0.03)$, total sleep time $(p<0.007)$, total wake time (time per minute $p<0.01)$, sleep efficiency $(p<0.01)$, light sleep percentage $(p<0.041)$, slow waves sleep percentage ( $p<$ $0.003)$ and on the sub type A1 index of the cyclic alternant pattern CAP $(p=-01)$ (Figures 2 and 3) (Table 4).

Despite the lack of significant differences concerning the number of awakenings, it is noteworthy to indicate that the duration of awakenings in group B were longer and with a frequent relationship with the appearance of electroencephalographic abnormalities.

\section{Discussion}

Most of the studied patients, previously diagnosed with idiopathic insomnia, showed electroencephalographic alterations during sleep. These alterations have a close relationship with the increase in the number of awakenings as well as with the increase of the time that the patients remain alert during the awakening. Thus, these data suggest that a high proportion of patients diagnosed with idiopathic insomnia, in fact, present an alteration of sleep consecutive to an electroencephalographic abnormality.

Table 4. Polysomnographic data: shows means and standard error (SEM) of polysomnographic variables in both groups. Significance levels were report on those variables that differed.

\begin{tabular}{lccc}
\hline & Grop A & Grop B & sig \\
\hline SL min $( \pm$ SEM $)$ & $24.7(8)$ & $54(16)$ & 0.03 \\
TST min $( \pm$ SEM) & $392.3(35)$ & $311(28.9)$ & 0.007 \\
TWT\% $( \pm$ SEM $)$ & $19(45)$ & $345(3.7)$ & 0.01 \\
WASO min $( \pm$ SEM) & $524(31)$ & $105.3(34)$ & \\
REML min $( \pm$ SEM) & $126(29)$ & $116.2(31)$ & \\
LS\% $( \pm$ SEM $)$ & $71.5(3.4)$ & $635(4.8)$ & 0.003 \\
SWS\% $( \pm$ SEM) & $110(3.0)$ & $207.3(3.6)$ & 0.041 \\
REM\% $( \pm$ SEM) & $17.2(2.2)$ & $15.7(2.2)$ & \\
Awakenings num. $( \pm$ SEM) & $7.4(3.7)$ & $19(109)$ & \\
Arousal Index $( \pm$ SEM) & $16.5(5.0)$ & $8.1(2.8)$ & \\
SE $( \pm$ SEM $)$ & $80.7(7.1)$ & $65.7(6.5)$ & 0.018 \\
CAP Index A1 $( \pm$ SEM) & $8.9(1.6)$ & $25.0(3.1)$ & 0.01 \\
CAP Index A2 $( \pm$ SEM) & $2.3(0.9)$ & $6.5(2.1)$ & \\
CAP Index A3 $( \pm$ SEM) & $13.1(3.8)$ & $9.0(2.6)$ & \\
\hline
\end{tabular}




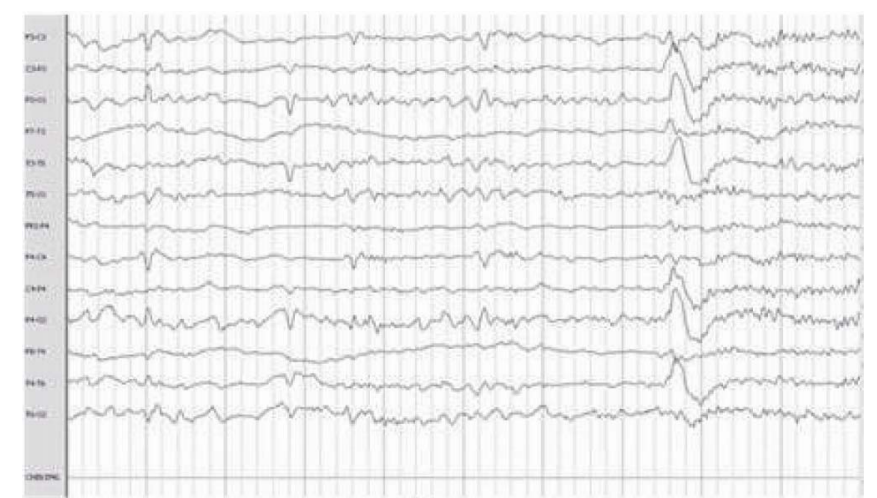

(a)

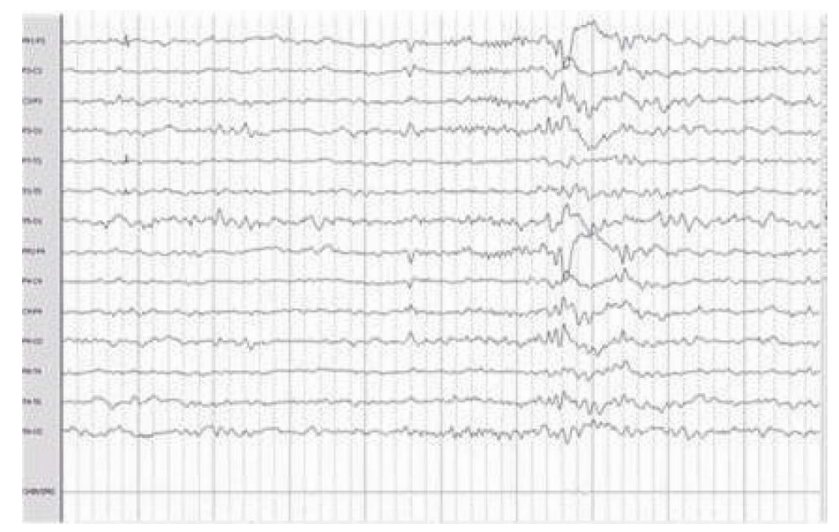

(c)

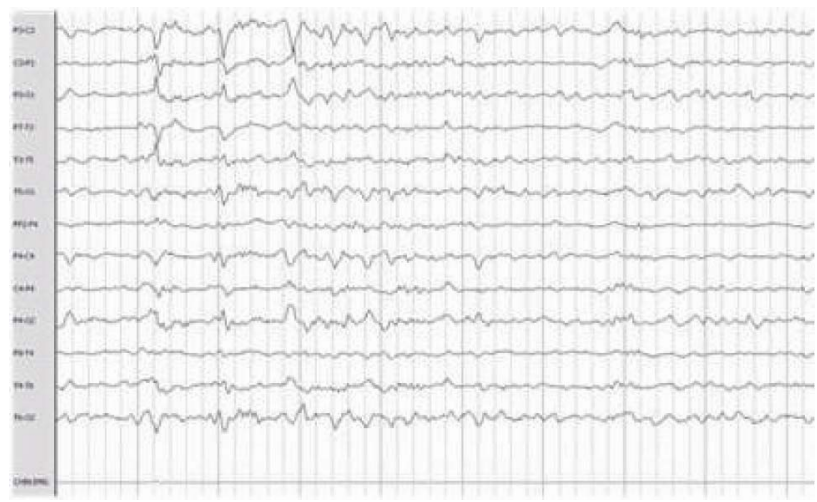

(e)

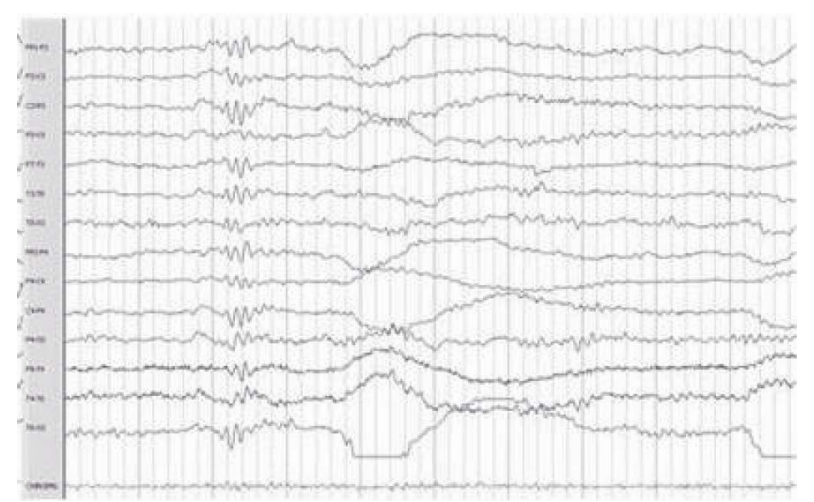

(b)

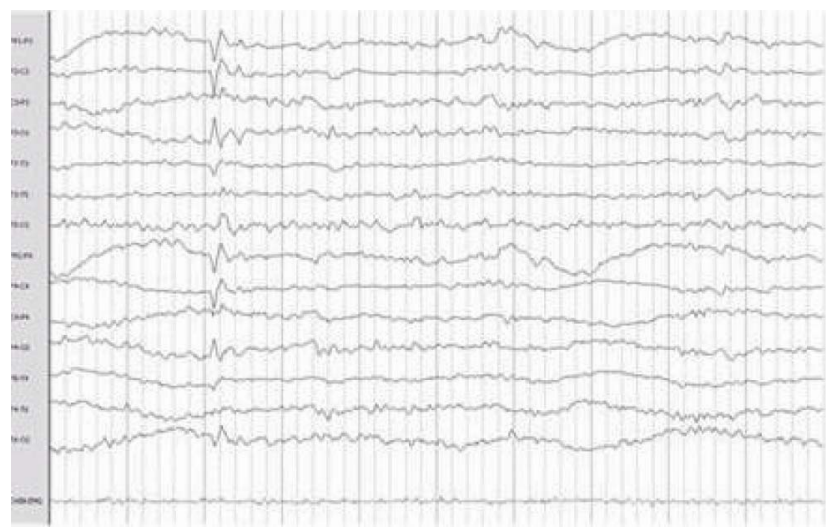

(d)

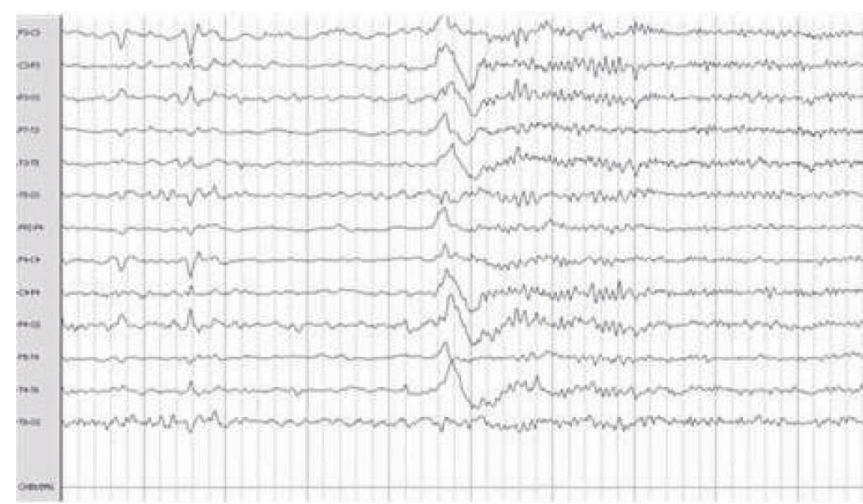

(f)

Figure 1. Electroencephalographic abnormalities: shows Frontal Spike (a), (f) Sharp waves paroxysm (b) Left frontal Spike (c) Bilateral fronto-central (d) Fronto-central spikes (e).

Supporting this idea, it is noteworthy to point out that patients diagnosed with idiopathic insomniac do not significantly increase their total sleep time using conventional treatment, as it was reported by authors using cognitive behavior therapy [19]. This resistance to this kind of treatment may be a result of brain malfunctioning associated to this kind of electrical alterations.

Although all the patients included in this study were diagnosed using the conventional clinical tools as idio- pathic insomniacs, PSG data indicates that they can be divided in two groups that are polysomnographically different. Group A, in which no electroencephalographic alterations were detected, showed the expected PSG variables such as increase of sleep latency, number and duration of awakenings, total wake time, among others parameters. On the other hand, group B showed altera tions of the same parameters, but the severity was significantly different when compared with group A. 
Total Sleep Time

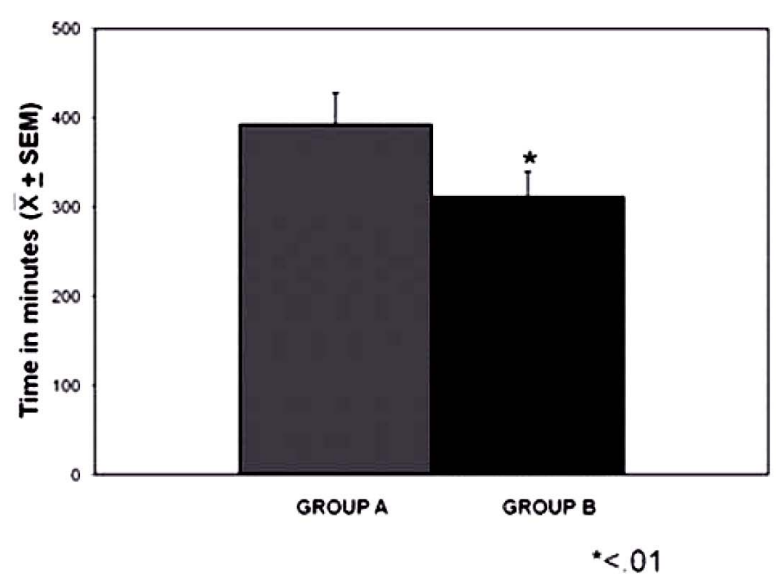

Total Wake Time

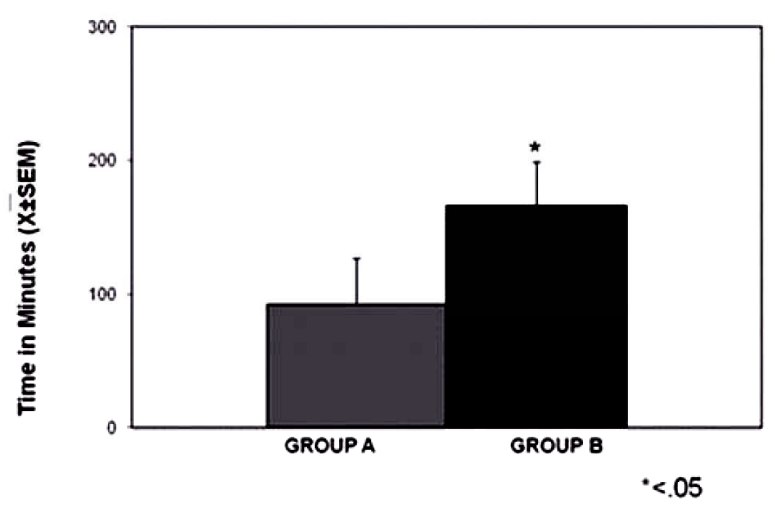

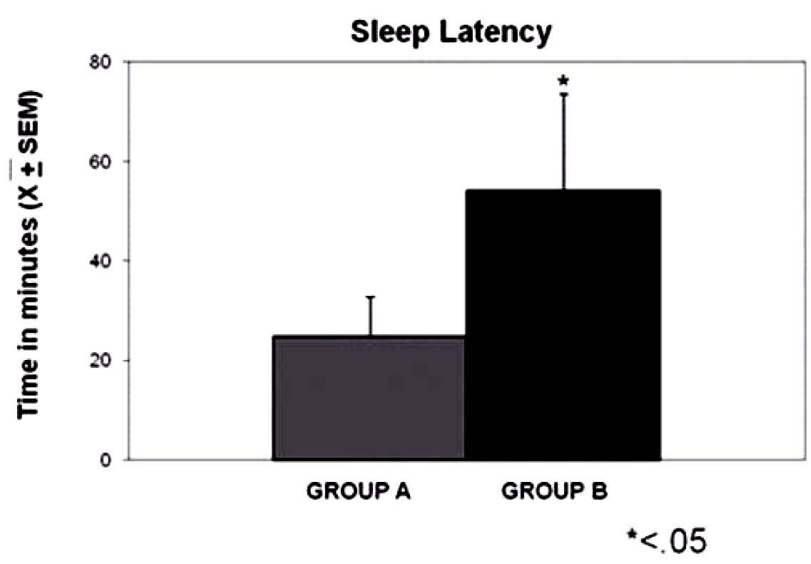

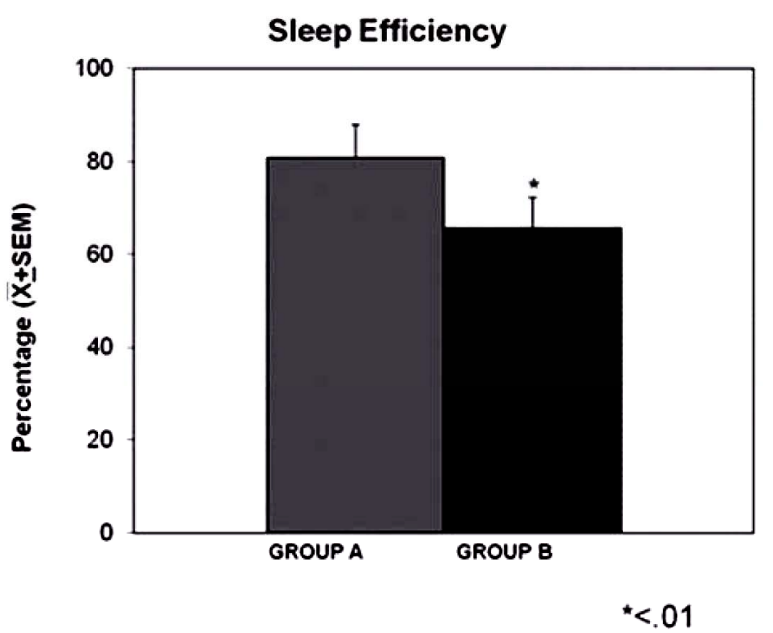

Figure 2. Polysomnographic data: shows polysomnographic variables significant differences were observed.

The present results were compared with those reported for idiopathic insomniacs by two different groups [8,9]. When our whole population data was compared, no significant differences were detected. However, when only our group B was compared several significant differences were detected. Concerning the study of Lafranchi our population with electroencephalographic alteration showed significant differences in TST, SE and SWS percentage. When the group was compared with the data of Jimenez Correa et al., significant differences were detected concerning TST, SE, SL and TWT (Table 5).

In addition, group A displays all the clinical signs already described for idiopathic insomniac patients (increase of sleep latency, wakefulness, awakenings number, as well as a decrease in total sleep time, sleep efficiency, among others), whereas the group B patients displays the same picture, but when the level of alteration was compared between groups, group B showed a significantly worsening of the symptoms (see Table 4).
Data not included in the present analysis indicate that patients in Group B (with electroencephalographic alterations) seek for medical help systematically sooner that the patients without electroencephalographic alterations. The present data suggest that this could be due to the fact that the severity of insomnia is significantly higher when the patients have an electroencephalographic alteration.

To further support the notion that we are facing actually two groups with a different illness, it must be mention that differences in gender were also observed. While group A showed a gender proportions according to the literature for idiopathic insomnia (1.5:1 women) [20,21], in group B, the proportion is different, with no prevalence of any gender $(1: 1)$. In addition, the manifestations of insomnia in the patients with no electroencephalographic abnormalities start early in life roughly during adolescence (mean 16 years old), while the patients with electroencephalographic alterations start a little bit later in life, about the 20 years of age (mean 22 years). Actually a 
Table 5. Mean difference: shows differences between ours groups and two different studies with idiopathic insomnia patient. Group B presents differences in sleep latency, total sleep time, sleep efficiency, slow wave sleep and total wake time in one or both studies, while group A do not presented those differences.

\begin{tabular}{|c|c|c|c|c|c|c|}
\hline & $\begin{array}{c}\text { Group A } \\
\mathrm{N}=10\end{array}$ & $\begin{array}{l}\text { Group B } \\
\mathrm{N}=12\end{array}$ & $\begin{array}{c}\text { Lanfranchi } 2009 \\
\mathrm{~N}=13\end{array}$ & $\begin{array}{l}\text { Group Band } \\
\text { Lanfranchi P }\end{array}$ & $\begin{array}{c}\text { Jimenez U. } 2007 \\
\mathrm{~N}=33\end{array}$ & $\begin{array}{c}\text { Group Band } \\
\text { Jimenez P }\end{array}$ \\
\hline TST min. & 392.3 & 311 & 411 & 0.000 & 382.8 & 0.001 \\
\hline SE (\%) & 80.7 & 65.7 & 85 & 0.000 & 78.8 & 0.006 \\
\hline SWS (\%) & 11 & 20.7 & 8 & 0.000 & 7.37 & 0.000 \\
\hline TWT (\%) & 19 & 34.5 & - & - & 23.1 & 0.011 \\
\hline SL min. & 24.7 & 54 & - & - & 19.6 & 0.011 \\
\hline
\end{tabular}

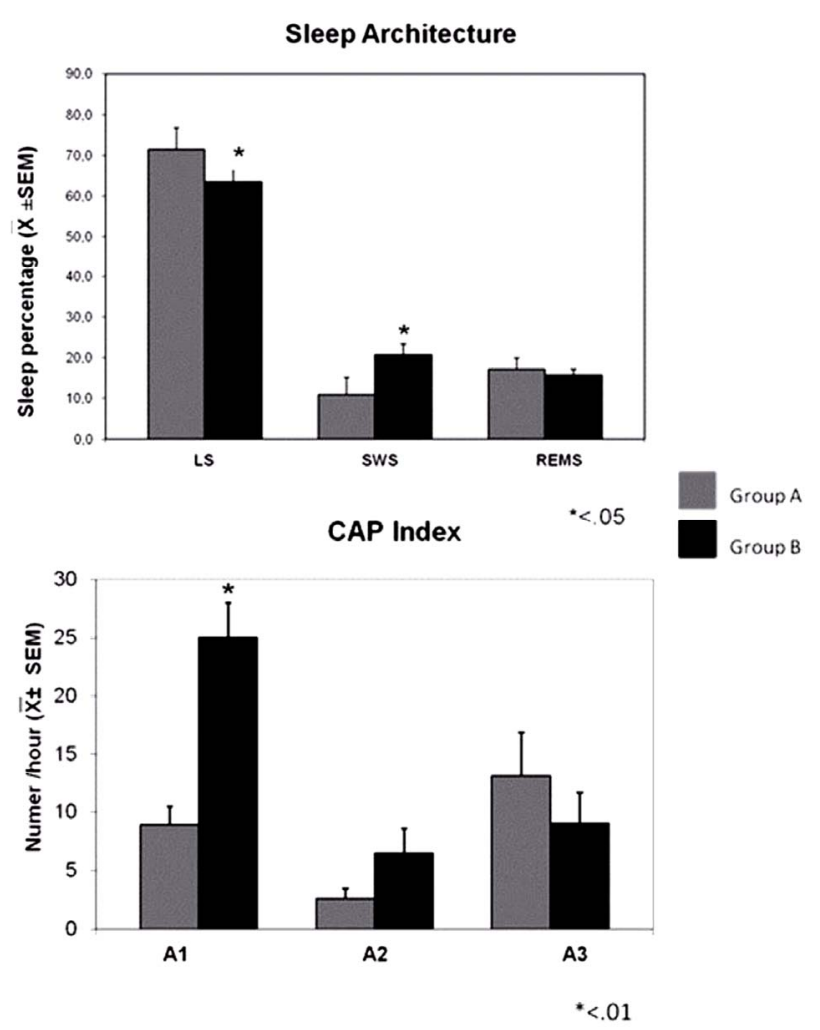

Figure 3. Sleep architecture and Cyclic Alternating Pattern: (up) shows sleep stages (LS, SWS and REM) in both groups. Group B has less light sleep and more slow wave sleep that group A. (Down) its represented CAP subtype; subtype A1 is significantly higher in group $B$.

significant positive correlation was observed between age of onset of insomnia and sleep efficiency.

\section{Conclusions}

The present data strongly suggest that within the population diagnosed with idiopathic insomnia, there is a subgroup in which the electroencephalographic alterations may play a critical role as the source of the sleep abnormality. Therefore, this subgroup should be diagnosed not with idiopathic insomnia but with insomnia related to electroencephalographic abnormalities. Of course, it is mandatory to determine the cause of such electroence- phalographic abnormalities; however, this kind of studies will be performed in the near future. The importance of this differential diagnosis lays on the more educated and more efficient treatment for each case. Furthermore, these data also supports the idea that, in some forms of insomnia, it is convenient to perform a complete EEG recording and not only the standard set usually establish in a polysomnographic study.

\section{Acknowledgements}

The study was partly supported by CONACYT Grant \# 257643 (DYAL). This study is part of the doctoral degree in biological sciences of DYAL at the Universidad Autónoma Metropolitana.

\section{REFERENCES}

[1] J. Edinger, R. Bootzin, D. Buysse, C. Espie, L. Lack, C. Morin, D. Riemann, A. Spilman, E. Stepanski and W. Insomnia, "The International Classification of Sleep Disorders. Diagnostic and Coding Manual," 2nd Edition, Westchester, Illinois, 2005. pp. 1-31.

[2] D. J. Taylor, K. L. Lichstein and H. H. Durrence, "Insomnia as a Health Risk Factor,” Behavioral Sleep Medicine, Vol. 1, No. 4, 2003, pp. 227-247. doi:10.1207/S15402010BSM0104_5

[3] M. M. Weissman, S. Greenwald, G. Niño-Murcia and W. C. Dement, "The Morbidity of Insomnia Uncomplicated by Psychiatric Disorders," General Hospital Psychiatry, Vol. 19, No. 4, 1997, pp. 245-250. doi:10.1016/S0163-8343(97)00056-X

[4] Z. De Saint Hilaire, J. Straub and A. Pelissolo, "Temperament and Character in Primary Insomnia," European Psychiatry, Vol. 20, No. 2, 2005, pp. 188-192. doi:10.1016/j.eurpsy.2004.04.009

[5] The American Psychiatric Association (APA), "Diagnostic and Statistical Manual of Mental Disorders," The American Psychiatric Association, 1980.

[6] M. G. Terzano, L. Parrino, M. C. Spaggiari, V. Palomba, M. Rossi and A. Smerieri, "CAP Variables and Arousals as Sleep Electroencephalogram Markers for Primary Insomnia," Clinical Neurophysiology, Vol. 114, No. 9, 2003, pp. 1715-1723.

[7] M. H. Bonnet and D. L. Arand, "The Consequences of a Week of Insomnia," Sleep, Vol. 19, No. 6, 1996, pp. 453- 
461.

[8] P. A. Lanfranchi, M. H. Pennestri, L. Fradette, M. Dumont, C. M. Morin and J. Montplaisir, "Nighttime Blood Pressure in Normotensive Subjects with Chronic Insomnia: Implications for Cardiovascular Risk," Sleep, Vol. 32, No. 6, 2009, pp. 760-766.

[9] Jimenez Correa U, Personal Communication, 2008.

[10] M. Terzano, L. Parrino, A. Smerieria, R. Chervinb, S. Chokrovertyc, C. Guilleminaut, et al., "Atlas, Rules, And Recording Techniques for the Scoring of Cyclic Alternating Pattern (CAP) in Human Sleep," Sleep Medicine, Vol. 3, No. 2, 2002, pp. 187-199. doi:10.1016/S1389-9457(02)00003-5

[11] M. Terzano, L. Parrino, M. Sapaggiari, V. Palomba, M. Rossi and A. Smerieri, "CAP Variables and Arousals as Sleep Electroencephalogram Markers for Primary Insomnia," Clinical Neurophysiology, Vol. 114, No. 9, 2003, pp. 1715-1723.

[12] Standards of Practice Committee of the American, "Sleep Disorders Association Practice Parameters for the Use of Polysomnography in the Evaluation of Insomnia," 1995.

[13] D. J. Buysse, A. Germain, M. L. Hall, D. E. Moul, E. A. Nofzinger, A. Begley, C. L. Ehlers, W. Thompson and D. J. Kupfer, "EEG Spectral Analysis in Primary Insomnia: NREM Period Effects and Sex Differences," Sleep,; Vol. 31, No. 12, 1 December 2008, pp. 1673-1682.

[14] M. L. Perlis, H. Merica, M. T. Smith and D. E. Giles, "Beta EEG Activity and Insomnia," Sleep Medicine Reviews, Vol. 5, No. 5, 2001, pp. 365-376. doi:10.1053/smrv.2001.0151
[15] K. Adam, M. Tomeny and I. Oswald, "Physiological and Psychological Differences between Good and Poor Sleepers," Journal of Psychiatric Research, Vol. 20, No. 4, 1986, pp. 301-316. doi:10.1016/0022-3956(86)90033-6

[16] E. A. Nofzinger, D. J. Buysse, A. Germain, et al., "Functional Neuroimaging Evidence for Hyperarousal in Insomnia," The American Journal of Psychiatry, Vol. 161, No. 11, 2004, pp. 2126-2128. doi:10.1176/appi.ajp.161.11.2126

[17] A. Rechtschaffen and A. Kales, "A Manual of Standardized Terminology: Techniques and Scoring System of Sleep Stages of Human Subjects," UCLA Brain Information Service/Brain Research Institute, Los Angeles, California, 1968.

[18] The American Academy of Sleep Medicine, "Rules, Terminology and Technical Specifications," Westchester, 2007.

[19] L. Van Houdenhove, B. Buyse, L. Gabriëls, I. Van Diest and O. Van Den Bergh, "Cognitive-Behavioral Therapy for Primary Insomnia: Effectiveness in a Clinical Setting," Tijdschrift voor Psychiatrie, Vol. 52, No. 2, 2010, pp. 79 88.

[20] D. J. Buysse and C. F. y Reynolds, "Insomnia," In: M. J. Thorpy, Ed., Handbook of Sleep Disorders, Marcel Dekker Inc., New York, 1990, pp. 373-434.

[21] M. E. Klink, S. F. Quan, W. T. Kaltembom and M. D. Lebowitz, "Risk Factor Associated with Complaints of Insomnia in a General Adult Population. Influence of Previous Complaints of Insomnia," Archives of Internal Medicine, Vol. 152, No. 8, 1992, pp. 1634-1637. doi:10.1001/archinte.152.8.1634 